7 Ascherio A. Epstein-Barr virus in the development of multiple sclerosis. Expert Rev Neurother 2008; 8: 331-333.

8 Grant WB, Holick MF. Benefits and requirements of vitamin D for optimal health: a review. Altern Med Rev 2005; 10: 94-111.

9 Thacker EL, Mirzaei F, Ascherio A. Infectious mononucleosis and risk for multiple sclerosis: a meta-analysis. Ann Neurol 2006; 59: 499-503.

10 Douglas AS, Brown T, Reid D. Infectious mononucleosis and Hodgkin's disease - a similar seasonality. Leuk Lymphoma 1996; 23: 323-331.

DOI: $10.1183 / 09031936.00098608$

\section{From the authors:}

We would like to thank A.W.J. Bossink and colleagues for their comments.

With regard to Epstein-Barr virus (EBV) serostatus, our laboratory has a diagnostic interest in EBV serology and our experience would indicate that for the age ranges in our published study [1] (i.e. both patients and controls) there will be $100 \%$ seropositivity for EBV. In Northern Ireland (UK), $60 \%$ of $10 \mathrm{yr}$ olds are seropositive; in adults, this figure rises to $>95 \%$ (unpublished data; P.V. Coyle, Regional Virus Laboratory, Belfast Health and Social Care Trust, Belfast, UK; personal communication).

Regarding the possible contamination of sputum samples with EBV DNA present in saliva, we have found that there is good correlation between EBV in lower and upper respiratory tract specimens, which is not consistent with contamination. We and other labs see little evidence of latent EBV when testing whole blood or white blood cells, making the presence of Bcells in sputum unlikely to be a confounding factor.

On the point of PCR being unable to differentiate between DNA derived from active viral replication or latent infection, as we were not detecting latency in B-cells (as previously noted) and as EBV causes a productive infection in epithelial cells, it follows current knowledge $[2,3]$ that we were measuring active viral replication. This also makes the determination of the number of B-cells in the sputum irrelevant.
The final issue regarding potential dilution of specimens with hypertonic saline is unlikely to be significant as previously published work has shown that induced sputum separated from saliva is similar to lower respiratory secretions expectorated spontaneously [4]. In the setting of our previously published study [1], any dilutional effect was negligible within the context of an assay determining viral load over six logarithms.

We would also like thank W.B. Grant for his comments in relation to vitamin $\mathrm{D}$ and chronic obstructive pulmonary disease. These comments raise several interesting points in this area and highlight the need for further prospective studies in this group of patients.

\section{T.E. McManus*, S. Elborn ${ }^{\#}$, H.J. O’Neill ${ }^{\uparrow}$, P.V. Coyle ${ }^{\Uparrow}$ and J. Kidney*}

*Dept of Respiratory Medicine, Mater Hospital, "Regional Adult Cystic Fibrosis Unit, Belfast City Hospital, and "Regional Virus Laboratory, Royal Victoria Hospital, Belfast, UK.

\section{STATEMENT OF INTEREST}

None declared.

\section{REFERENCES}

1 McManus TE, Marley A-M, Baxter N, et al. High levels of Epstein-Barr virus in COPD. Eur Respir J 2008; 31: 1221-1226.

2 Feederle R, Neuhierl B, Bannert H, Geletneky K, ShannonLowe C, Delecluse HJ. Epstein-Barr virus B95.8 produced in 293 cells shows marked tropism for differentiated primary epithelial cells and reveals interindividual variation in susceptibility to viral infection. Int J Cancer 2007; 121: 588-594.

3 Shannon-Lowe CD, Neuhierl B, Baldwin G, Rickinson AB, Delecluse HJ. Resting B cells as a transfer vehicle for Epstein-Barr virus infection of epithelial cells. Proc Natl Acad Sci USA 2006; 103: 7065-7070.

4 Pizzichini MM, Popov TA, EfthimiadisA, et al. Spontaneous and induced sputum to measure indices of airway inflammation in asthma. Am J Respir Crit Care Med 1996; 154: 866-869.

\title{
Extensively drug-resistant tuberculosis: is its definition correct?
}

\section{To the Editors:}

Two articles by MigLIORI and co-workers [1, 2], which recently appeared in the European Respiratory Journal (ERJ), may support the idea that the current definition of extremely drug-resistant tuberculosis (XDR-TB) [3, 4] is not the most adequate.
In recent years, $\mathrm{XDR}$-TB has become a major concern as it leads to incurable TB in a significant proportion of patients $[4,5]$. XDR-TB was first defined in March 2006 as multidrug-resistant TB (MDR-TB; resistance to isoniazid and rifampicin) plus resistance to at least three of the six second-line anti-TB drug groups (fluoroquinolones, aminoglycosides, polypeptides, 
thioamides, cycloserine and para-aminosalicylic acid (PAS)) [6]. However, this definition permits the possibility of susceptibility to fluoroquinolones and aminoglycosides (kanamycin, amikacin) and/or polypeptides (capreomycin) in some XDR-TB patients, meaning much higher success rates could be achieved if such drugs were used. Amongst second-line drugs, only the fluoroquinolones and injectables (aminoglycosides and polypeptides) have bactericidal activity and could be considered very effective. Fluoroquinolones and injectables therefore seem to represent the same as isoniazid and rifampicin amongst the first-line drugs. Acknowledgement of the fact that the success of treatment with second-line drugs depends on the use of fluoroquinolones and injectables (aminoglycosides and polypeptides), in addition to the fact that susceptibility testing to these drugs produces more reliable and reproducible results, prompted a modification of the definition of XDR-TB. Currently, XDR-TB is defined as MDR-TB plus resistance to fluoroquinolones and to at least to one of the second-line injectables (kanamycin, amikacin and capreomycin) $[3,4]$.

Although two recent studies have shown that the current definition of XDR-TB is predictive of a poorer clinical outcome than MDR-TB [1,7], this definition may still be inappropriate even though it is clearly better than the first. This is particularly so because, in special cases, it allows the use of some first-line drugs and/or one of the injectables (kanamycin, amikacin or capreomycin).

The current definition permits the possibility of susceptibility to ethambutol and/or pyrazinamide, which, although rare, could be present in any given case. In a recent study, MigLIORI et al. [1] presented the unfavourable outcome of MDR-TB cases resistant to all first-line drugs compared with other MDR-TB cases in which there was susceptibility to ethambutol, pyrazinamide or streptomycin. Table 1 shows that the role of pyrazinamide was avery important in three studies and that a success rate of $>90 \%$ was achieved with pyrazinamide plus ethionamide and cycloserine [8-10, 15].

The current XDR-TB definition also allows the use of one of the injectables (kanamycin, amikacin or capreomycin) along with ethionamide, cycloserine and PAS, thereby reaching a possible cure rate of $>80 \%$ [15], as can be observed in four of the studies presented in table 1 [11-14]. The possibility of success in an XDR-TB patient using an injectable and all of the second-line drugs could therefore be very close to that achieved in patients with MDR-TB and without XDR-TB (for example, in MDR-TB patients with susceptibility to all of the injectables) [15]. In their more recent study, MigLIORI et al. [2] evidenced the favourable outcome of XDR-TB patients with susceptibility to capreomycin, the least frequently used injectable in the world, and, for this reason, susceptibility is possible in many patients with resistance to kanamicyin and/or amikacyn.

For all of these reasons, the most accurate definition of extensively drug-resistant tuberculosis would be cases with resistance to all first-line drugs (not only those defining multidrug-resistant tuberculosis) and to fluoroquinolones and all of the injectables (not just to one), the two most potent secondline drugs groups. Usually, these patients have a possible treatment success rate of $<50 \%$ and clearly stand apart from exclusively multidrug-resistant tuberculosis patients. If the current extensively drug-resistant tuberculosis definition is maintained, we will shortly be in need of a new definition for those cases, thus constituting a new level of difficulty in antituberculosis treatment for which the best classification would be XXDR (extensively extensively drug-resistant tuberculosis).

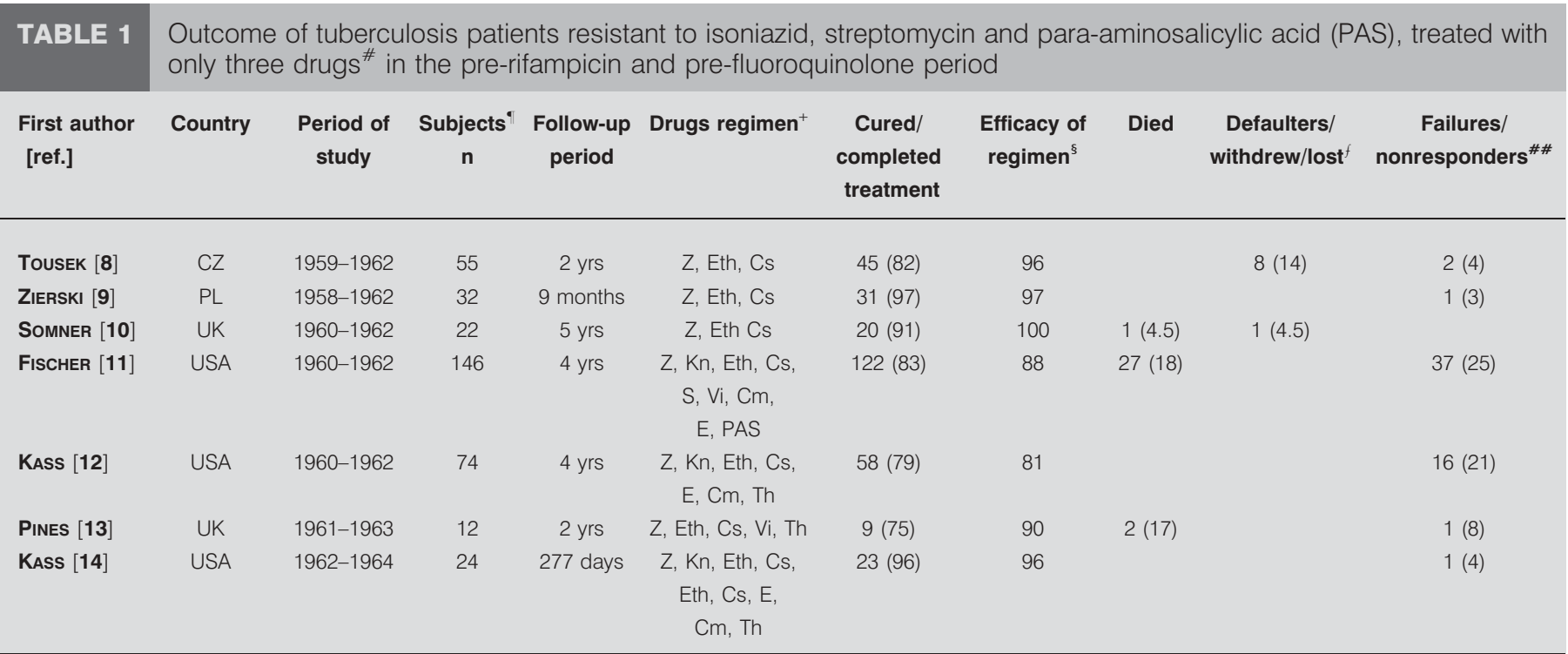

Data presented as $\mathrm{n}(\%)$ or \%, unless otherwise stated. Z: pyrazinamide; Eth: ethionamide; Cs: cycloserine; Kn: kanamycin; S: Streptomycin; Vi: viomycin;

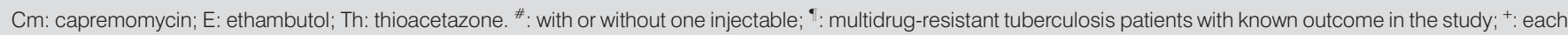
subject took only three of these drugs; ${ }^{\text {s: }}$ those who were cured/completed treatment divided by those who were cured/completed treatment plus failures/nonresponders; $f$ : patients not taking drugs regularly are included; ${ }^{\# \#}$ : including relapses known in patients cured previously. Reproduced and modified from [15] with permission from the publisher. 


\section{J.A. Caminero}

Pneumology Dept, General Hospital of Gran Canaria “Dr. Negrín", Barranco de la Ballena s/n, Las Palmas, Spain.

\section{STATEMENT OF INTEREST}

None declared.

\section{REFERENCES}

1 Migliori GB, Besozzi G, Girardi E, et al. Clinical and operational value of the extensively drug-resistant tuberculosis definition. Eur Respir J 2007; 30: 623-626.

2 Migliori GB, Lange C, Centis R, et al. Resistance to secondline injectables and treatment outcomes in multidrugresistant and extensively drug-resistant tuberculosis cases. Eur Respir J 2008; 31: 1155-1159.

3 World Health Organization. The global MDR-TB \& XDRTB response plan 2007-2008. Geneva, World Health Organization, 2007.

4 Raviglione MC, Smith IM. XDR tuberculosis - implications for global public health. N Engl J Med 2007; 356: 656-659.

5 Gandhi NR, Moll A, Sturm AW, et al. Extensively drugresistant tuberculosis as a cause of death in patients coinfected with tuberculosis in a rural area of South Africa. Lancet 2006; 368: 1575-1580.

6 Centers for Disease Control and Prevention. Emergence of Mycobacterium tuberculosis with extensive resistance to second-line drugs - worldwide, 2000-2004. Morb Mortal Wkly Rep 2006; 55: 301-305.

7 Jeon CY. Extensively drug-resistant tuberculosis in South Korea: risk factors and treatment outcome among patients at a tertiary referral hospital. Clin Infect Dis 2008; 46: 42-49.
8 Tousek J, Jancik E, Zelenka M, Jancikova-Máková M. The results of treatment in patients with cultures resistant to streptomycin, isoniazid and PAS, a five-year follow-up. Tubercle 1967; 48: 27-31.

9 Zierski M, Zachara A. Late results in re-treatment of patients with pulmonary tuberculosis. Tubercle 1970; 51: 172-177.

10 Somner AR, Brace AA. Late results of treatment of chronic drug-resistant pulmonary tuberculosis. BMJ 1966; 1: 775-778.

11 Fischer DA, Lester W, Dye WE, Moulding TS. Re-treatment of patients with isoniazid-resistant tuberculosis. Analysis and follow-up of 146 cases. Am Rev Respir Dis 1968; 97: 392-398.

12 Kass I. Chemotherapy regimens used in retreatment ofpulmonary tuberculosis. I. Observations on the efficacy of combinations of kanamycin, ethionamide and either cycloserine or pyrazinamide. Tubercle 1965; 46: 151-165.

13 Pines A. Treatment of pulmonary tuberculosis with cultures resistant to two or more drugs: a series of 44 patients. Tubercle 1965; 46: 131-142.

14 Kass I. Chemotherapy regiments used in retreatment of pulmonary tuberculosis. II. Observations on the efficacy of combinations of ethambutol, capreomycin and companion drugs, including 4-4 diisoamyloxythiosemicarbanilide. Tubercle 1965; 46: 166-177.

15 Caminero JA. Treatment of multidrug-resistant tuberculosis: evidence and controversies. Int J Tuberc Lung Dis 2006; 10: 829-837.

\section{An ecological analysis of incidence of tuberculosis and per capita gross domestic product}

\section{To the Editor:}

In 2006, an estimated 9 million new cases of tuberculosis (TB) emerged worldwide [1]. Of these, only $1 \%$ occurred in the European Union and North America combined, while Africa and South-East Asia contributed $>65 \%$. Among the 22 highburden countries, 17 were in Africa, 16 of which were in the lowest quartile in terms of per capita gross domestic product (GDP). Since 2001, the fight against poverty has been a major theme in the World Health Organization's (WHO) "Stop TB" strategy. Indeed, poverty "fuels" TB by facilitating transmission through crowded working and living conditions, it may increase the risk of progression to disease through malnutrition, and imposes barriers to accessing health services.

The aim of the present analysis was to explore and illustrate the relationship between the incidence of TB and an indicator of standard of living (per capita GDP) using WHO and World Bank estimates.
The per capita GDP, i.e. total market value of all final goods and services produced within a given country in a given period of time, is often used as an indicator of standard of living. The advantages are that it is measured frequently, widely used and easily accessible information, and that technical definitions for estimating GDP are relatively consistent between countries.

The World Bank publishes yearly estimates of per capita GDP per country [2]. Similarly, the WHO provides annual estimates for TB incidence for each country by WHO region [2]. Of the 211 WHO member states and 177 countries with reports by the World Bank, there were 171 pairs with information on both GDP and TB. For uniformity of the denominator, United Nations population estimates for 2004 [3] were used to obtain per capita figures for GDP and TB, respectively.

The choice of the same denominator permitted a direct regression on the numerators (rather than on rates), using population size as a weight. Linear regression on the logarithm 